\title{
Application of Entropy Weight Method in the Evaluation of the Smart Growth
}

\author{
Jiaxing Zhang \\ North China Electric Power University, Baoding 071000, China. \\ jiaxingzhang_ncepu@163.com
}

Keywords: evaluation metric, Entropy Method, NARX Neural Networks, smart growth.

\begin{abstract}
To make contributions to solve urban problems, we propose eight evaluation metric based on Smart Growth theory, and offer strategies to the smart growth of the selected cities. Firstly, considering the three E's of sustainability, the principles are divided into three aspects which are transportation, land uses, and community development. Secondly, eight factors are established. Then, with the city development data of the two cities in recent years, we establish the evaluation model via using entropy method. After that, the metric weight and comprehensive score is used to evaluate the success of city development.
\end{abstract}

\section{Introduction}

Living on building cities which is economically prosperous, socially equitable, and environmentally sustainable is a tireless dream. So that many communities are implementing smart growth initiatives in an effort to consider long range, sustainable planning goals. Smart growth [1]is an urban planning theory that originated in 1990's as a means to curb continued urban sprawl and reduce the loss of farmland surrounding urban centers. According to a large number of literature, the three E's of sustainability and the 10 principles of smart growth, the principle was divided into three aspects which is transportation, land uses, and community development. Taking Lijiang and Arlington for example, this paper makes a comparative analysis.

\section{Establishment and Solution of the Model}

\subsection{Establishment of Metric}

Through the analysis of the principles of smart growth, we find that the principles respectively from transportation, land use, and community development measure the development of the city. Besides these, some data in the National Statistical Yearbook are able to reflect the development of cities. So that, the below metric is put forward based on the analysis.

Per capita floor area

We define that the ratio of urban area to the population is used to evaluate the per capita floor area.

$$
F A_{p c}=\frac{C A_{\text {total }}}{P_{\text {total }}}
$$

\section{Variety of architecture types(VAT)}

We use the distribution density of various types of buildings in the city to measure the variety of architecture types.

$$
V A T=\frac{N M T B}{A S}
$$

\section{Per capita housing growth area}

We define the area of per capita housing growth as the ratio of new housing to the number of new population.

$$
H G A_{p c}=\frac{H G A_{\text {total }}}{P_{\text {total }}}
$$

Per capita green area 
We define the ratio of the total green area to the total population is

We need to define the per capita GDP growth rate as the ratio of the annual increase in GDP to the annual increase in population.

$$
r_{p c G D P}=\frac{p c G D P_{\text {now }}-p c G D P_{\text {last }}}{p c G D P_{\text {last }}} \times 100 \%
$$

\section{Gross National Happiness(GHN)}

Gross National Happiness is a measure of people's development of the feelings and experiences which reflects the core indicators of the quality of life of residents.

$$
G N H=\omega_{1} C_{1}+\omega_{2} C_{2}+\omega_{3} C_{3}+\omega_{4} C_{4}+\omega_{5} C_{5}+\omega_{6} C_{6}
$$

$C_{\mathrm{i}}(i=1,2,3 \ldots 6)$ : Index of various factors

$\omega_{i}(i=1,2,3 \ldots 6)$ : The weight of each parameter $C$

\section{Density of road network}

The ratio of total mileage to total area is designed to measure the density of road network.

$$
D R N=\frac{R L_{\text {total }}}{C A_{\text {total }}}
$$

\section{Model of traveling}

The available mode of transport is used to describe the model of traveling.

\subsection{The Metric Weight Based on Application of Entropy Method}

Entropy Method (EM) is a branch of information theory. It can capture the implied interactions among factors, and indicate the grade of each factor. Then we can determine the weight of each factor based on the relative size of its grade. In this model, we follow the steps below to calculate the weight of each factor.

\section{Step1: Standardization of data}

First of all, it is necessary for us to standardize the data, because the data of the metric index is not uniform and can't be processed. So that, the data will be standardized according to the following methods.

$$
x_{i j}{ }^{\prime}=\frac{x_{i j}-\min \left\{x_{j}\right\}}{\max \left\{x_{j}\right\}-\min \left\{x_{j}\right\}}
$$

On the contrary, for negative indicators such as per capita floor area. The smaller values reflect the higher efficiency of the urban land use, the denser urban residential and the better effect of the Smart Growth. So take the following formula for its standardization.

$$
x_{i j}{ }^{\prime}=\frac{\max \left\{x_{j}\right\}-x_{i j}}{\max \left\{x_{j}\right\}-\min \left\{x_{j}\right\}}
$$

Where $x_{i j}$ 'is the standardized data, $\min \left(x_{j}\right) / \max \left(x_{j}\right)$ is the minimum and maximum values, $x_{i j}{ }^{\prime}$ is the metric data. Through this operation, all of the data is in the $[0,1]$ range.

Step 2: Calculate entropy of information

Entropy of information is an important factor to measure the weight of evaluation index, the great entropy of information shows that the information provided by the index in the comprehensive score is large and the weight is higher. The equation below indicates how to calculate entropy of information.

$$
E_{\mathrm{j}}=-\ln (\mathrm{n})^{-1} \sum_{\mathrm{i}=1}^{\mathrm{n}} x_{i j}{ }^{\prime} \ln x_{i j}{ }^{\prime}
$$

Where $n$ is the amount of the data, $x_{i j}$ ' is the standardized data.

\section{Step 3: The calculation of weight}

Having calculated entropy of Information, the weight of each index is determined by using the theory of entropy, which reflects the importance of the index in the comprehensive evaluation system. 


$$
W_{j}=\frac{1-E_{j}}{8-\sum E_{j}}, j=(1,2, \ldots, 8)
$$

According to the gathered data of the cities which is shown in the appendix, we are able to acquire the weight of every metric as the following table.

Step 4 : Comprehensive score

In terms of the weight, we can obtain the Comprehensive score.

$$
Z=\sum_{j=1}^{8} X^{\prime}{ }_{i j} W_{j}
$$

According to the metric model, combined with the above two cities over the years of development data, using EM analysis method, we can get the weight of each metric is as follows:

Table 1 The metric weight

\begin{tabular}{cc}
\hline Metric & Weight \\
\hline$F A_{p c}$ & 0.0764 \\
$V A T$ & 0.1378 \\
$H G A_{p c}$ & 0.1735 \\
$C G A_{p c}$ & 0.1424 \\
$r_{p c G D P}$ & 0.1550 \\
$G H N$ & 0.1195 \\
$D R N$ & 0.1056 \\
$M T$ & 0.0898 \\
\hline
\end{tabular}

\subsection{Application of Model}

With the Time Series NARX Feedback Neural Networks Forecast Model [2], we regard the data of city development for nearly ten years in Lijiang city and nearly eight years of Arlington as the system's internal input layer. Finally, the forecasting data of the eight indicators is obtained which is shown in the following table.

Table 2 Forecast results

\begin{tabular}{ccc}
\hline Metrics & Arlington & Gucheng \\
\hline$F A_{p c}$ & 308.0176 & 1.5038 \\
$V A T$ & 460.7982 & 148.3693 \\
$H G A_{p c}$ & 2.0778 & 1.3717 \\
$C G A_{p c}$ & 0.3583 & 0.3855 \\
$r_{p c G D P}$ & 0.0168 & 0.4107 \\
$G H N$ & 0.753 & 0.194 \\
$D R N$ & 63.3716 & 77.1057 \\
$M T$ & 84.3512 & 75.8482 \\
\hline
\end{tabular}

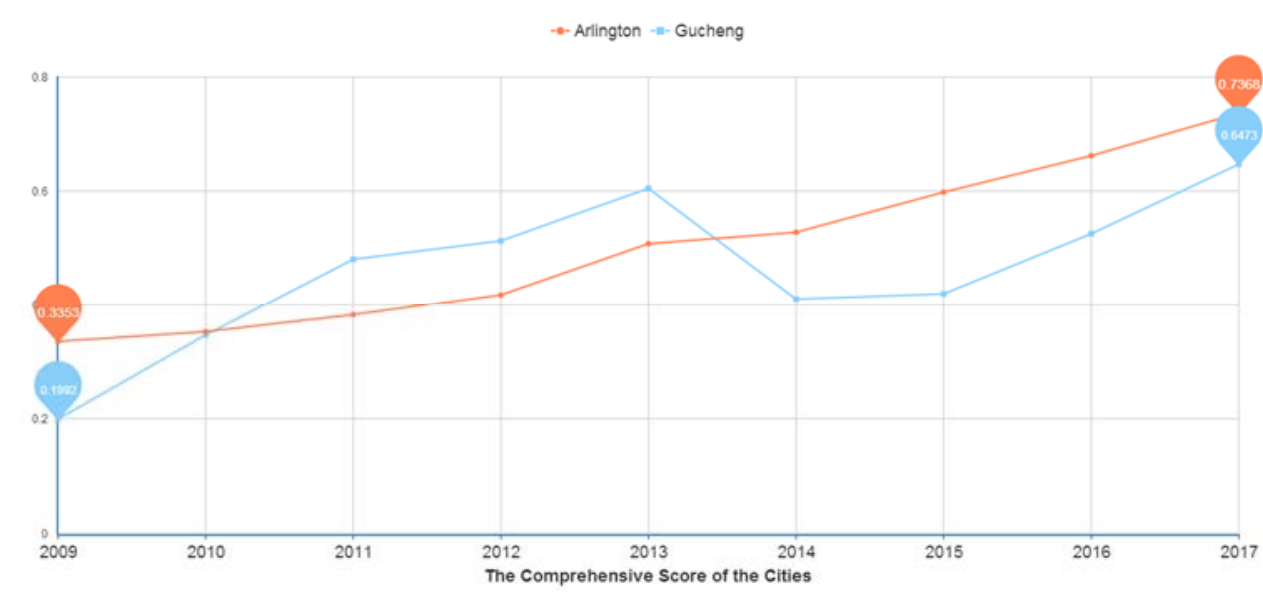

Fig. 1 Forecast comprehensive score 
As shown in Figure 1, the comprehensive score of the two city in 2017 compared with the previous is on the rise, which indicates the both cities is meet the smart growth. In addition, the comprehensive score is higher than the Old Town of Lijiang in Arlington as an example of the smart growth.

At the same time, in terms of the historical data, the growth in Lijiang is not stable enough. Therefore, the development plan did not fully realize the smart growth. On the contrary, the comprehensive score of Arlington increased steadily. Obviously the city achieves the goal of smart growth.

In conclusion, the selected cities are designed to achieve smart growth. However, the development of Arlington is more effective.

\section{References}

[1] Li Ming; Study on the Harmonious Community Construction and Evaluation Index System Based on the Principle of Smart Growth; Changan Univrsity, Xi.an, China.

[2] MathWorks: Design Time Series NARX Feedback Neural Networks Online:https://cn.mathworks.com/help/nnet/ug/design-time-series-narx-feedback-neuralnetworks.html?s_tid=srchtitle. 